\title{
Proposal of a Touch Panel Like Operation Method For Presentation with a Projector Using Laser Pointer
}

\author{
${\text { Yuya Kawahara, }{ }^{\mathrm{a},} \text { and Lifeng Zhang }}^{\mathrm{a}}$ \\ ${ }^{\mathrm{a} K y u s h u}$ Institute of Technology, \\ 1-1 Sensui-cho Tobata-ku, Kitakyushu city, Fukuoka-ken, 804-8550, Japan \\ *Corresponding Author: kawahara@boss.ecs.kyutech.ac.jp
}

\begin{abstract}
A presentation using a projector and a laser pointer is a very popular scene at a business meeting or at an academic conference. A system operate the mouse cursor by a laser pointer is proposed to perform more comfortable presentation. However, as for the proposed system using the image processing approach, the using environment is limited because of the frame rate, environment light problems. To wipe such a disadvantage, an additional operation tools equipped with an acceleration sensor that coordinate the movement of the cursor based on visual feedback are suggested. But this could make the usability spoiled if the presenter is not well trained. In this system, a modulated laser source is adopted to reduce the surrounding light noise, and the PSD sensor gives a very rapid response than a CCD camera system. In addition, we become able to operate it in real time because no image processing was used.
\end{abstract}

Keywords: PSD sensor, modulated laser, presentation.

\section{Introduction}

Presentations using a computer projector system are efficient way for one-way communication. It is performed in various scenes including the business meeting, the lecture and the class at the university and the company. In recent years, people use a laser pointer to point to the gazing point during the presentation time. Furthermore, various tools have been developed for making presentations more effectively and efficiently. In those systems, a "stick mouse" in which an acceleration sensor installed can operate the system by a swing of the hand is exists. However, the user may feel stress because such operations need to regulate the movements based on feedback by the sight through the presentation time. Besides, to operate the view screen, another system extracts the laser irradiation spot position by image processing is proposed. ${ }^{(1)(2)(3)}$ But the light of projector and the illumination of external light influence it easily, the using environment is limited. ${ }^{(4)(5)}$

Therefore, in this study, the proposed system performs an intuitive cursor operation by the laser pointer using PSD. In this system, we utilized a modulated laser source and there are no limits of the using environment because the modulated laser source can be separated well from ambient light. In addition, we become able to operate it in real time because we do not use image processing.

\section{Proposed System}

\subsection{Construction of proposed system}

In this study, we assume that a PC screen was projected to a view screen by a projector, and irradiate a light spot to the view screen by using the laser pointer in which installed a modulation function. This system first calculates the coordinate of the laser spot irradiated on the view screen, and then converts it to the PC screen coordinate. Finally, based on the calculated information, the light spot can be treated as a mouse cursor on the PC screen. Therefore, we can realize various operations by using a laser pointer.

The system implements two following functions in this study.

(a) Draw a picture on the PC screen by tracing a picture projected on the view screen using a laser light spot irradiated on screen

(b) Performs the touch panel like operation corresponding to a laser light spot irradiated on view screen 
Using these two functions, it is possible to write a memo timely on the view screen during the presentation or operate a PC with a touch panel-like operation by using a laser pointer. In addition, the presenter can operate it intuitively by chasing a laser light spot with eyes. Figure 1 shows the proposed system configuration and Fig.2 shows the flowchart of processing. This device is constructed by a laser pointer, a PSD sensor and a PC shown in Fig.1.

At first a frequency modulation circuit produces an modulated power source and supplies it to a laser. When the user irradiates the laser point on screen, the PSD receives the reflected light through a lens. Because the PSD is a light responding device, the excited signal in which include the surrounding noise is input into the circuit of the light-receiving department. In this research, through a band pass filter, the extra noise is removed. After D/A convert the extracted signal, the digitalized signal is inputted into a $\mathrm{PC}$ and the coordinates of the laser point are figured out. In this way, the system replaces a calculated coordinate of view screen with the coordinate of the PC screen and moves mouse cursor to the coordinate on the PC screen.

\subsection{The two-dimensional coordinate detection using the PSD}

In order to measure the two-dimensional coordinates of the laser point, a two-dimensional position sensitive detector (PSD) shown in Fig. 3 is used.

The PSD is a kind of optical sensor using the $\mathrm{Si}$ photodiode. PSD can detect the position of the gravity center of the total irradiation light. The feature of PSD is high sensitivity, and could detect the position with a high

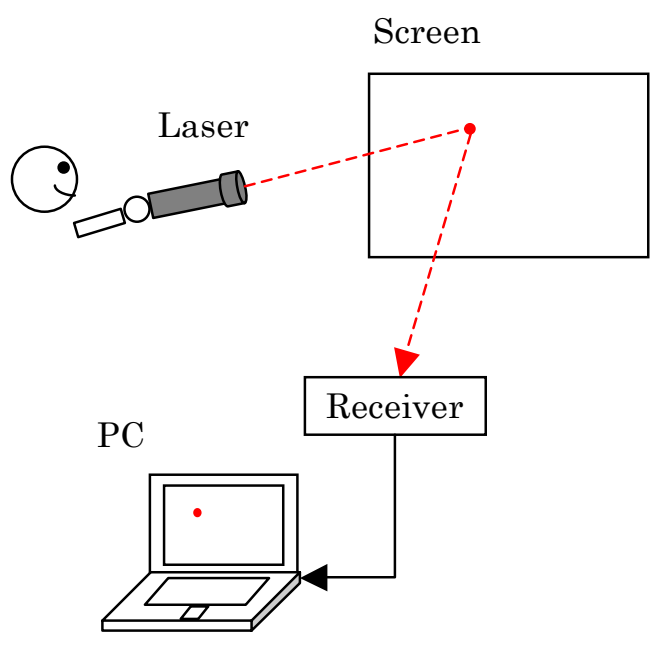

Fig. 1. System Configuration. resolution and a fast response.

The PSD generates the electro charge according to the amount of light reaching a light-receiving surface. The PSD has 4 terminals corresponding to the $\mathrm{X}-\mathrm{Y}$ axis. For these terminals, the resistance values from the light-receiving point to each terminal vary according to the lighted position. That is, the output currents from 4 terminals are also changing by the lighted position. By using the ration calculated by the currents from 4 terminals, the position of the light spot can be detected as the gravity of the light spot.

In this study, we use the 2D PSD sensor S2044, which manufactured by Hamamatsu Photonics. Its active area is $4.7 \times 4.7 \mathrm{~mm}$.

In Fig.3, we show coordinates of the center-of-gravity point as $(x, y)$. According to the center-of-gravity point of this spot light, current value $I_{X 1}, I_{X 2}, I_{Y 1}$, and $I_{Y 2}$ are obtained from the electrode of the four electrodes $X 1, X 2, Y 1$, and $Y 2$, respectively. In this case, the $X$ coordinate of the center-of-gravity is given as the following equation.

$$
\frac{\left(I_{X 2}+I_{Y 1}\right)-\left(I_{X 1}+I_{Y 2}\right)}{I_{X 1}+I_{X 2}+I_{Y 1}+I_{Y 2}}=\frac{2 x}{L_{x}}
$$

The following equation calculates the $\mathrm{Y}$ coordinate of the center of gravity.

$$
\frac{\left(I_{X 2}+I_{Y 2}\right)-\left(I_{X 1}+I_{Y 1}\right)}{I_{X 1}+I_{X 2}+I_{Y 1}+I_{Y 2}}=\frac{2 y}{L_{y}}
$$

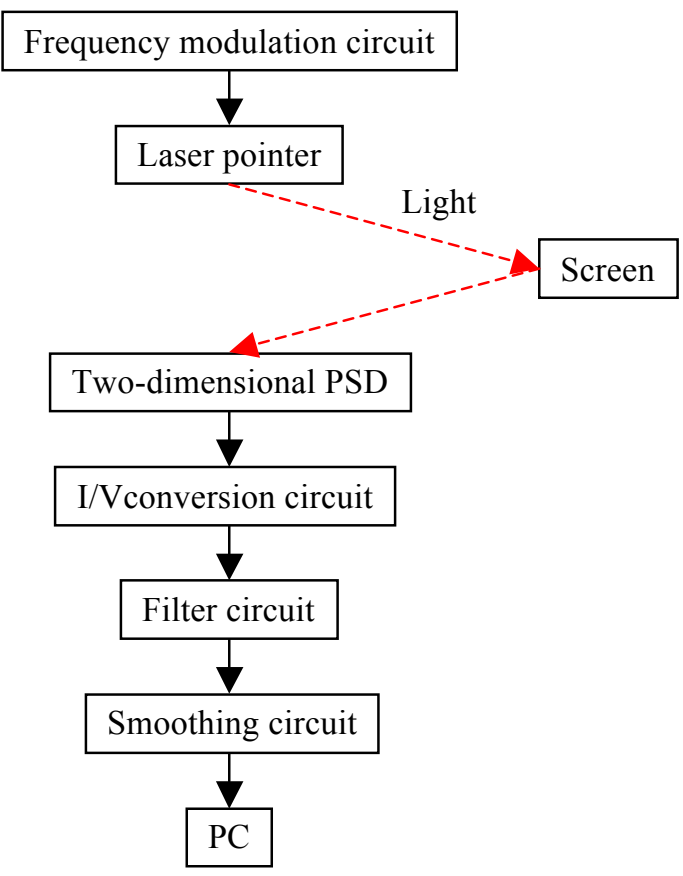

Fig. 2. Flowchart. 
Moreover, since $L_{X}$ and $L_{Y}$ are $L_{X}=5.7 \mathrm{~mm}$ and $L Y=$ $5.7 \mathrm{~mm}$ in S2044 respectively, the center-of-gravity of laser light spot can be calculated by the conversion equations (1) and (2).

The light of the laser spot on view screen is focused on the surface of PSD through the lens. Figure 4 shows this relationship. Figure 5 shows $\mathrm{OG}_{\mathrm{x}} \mathrm{AO}^{\prime} \mathrm{G}_{\mathrm{x}}{ }^{\prime}$ plane of Fig.4. Since the value $z$ and $D$ is constant in Fig.5. $X$ is calculated

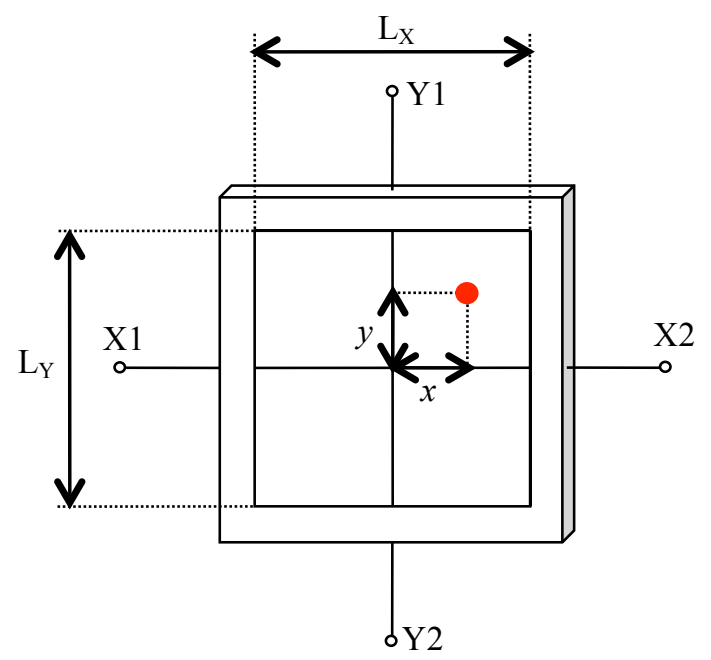

Fig. 3. 2 Dimensional PSD.

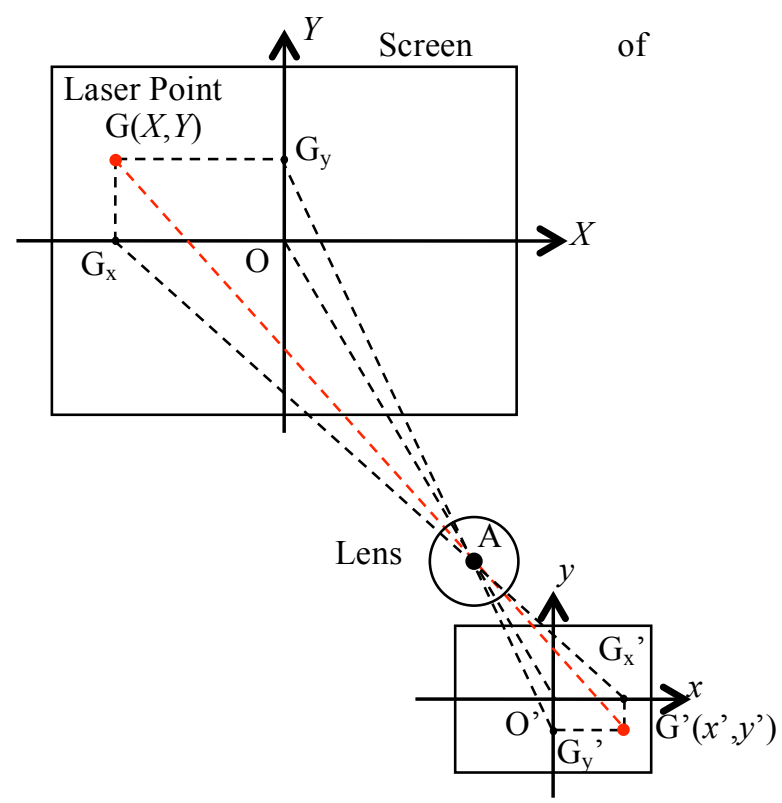

Light-Receiving Surface of the PSD

Fig. 4. State of the Light Receiving of PSD.

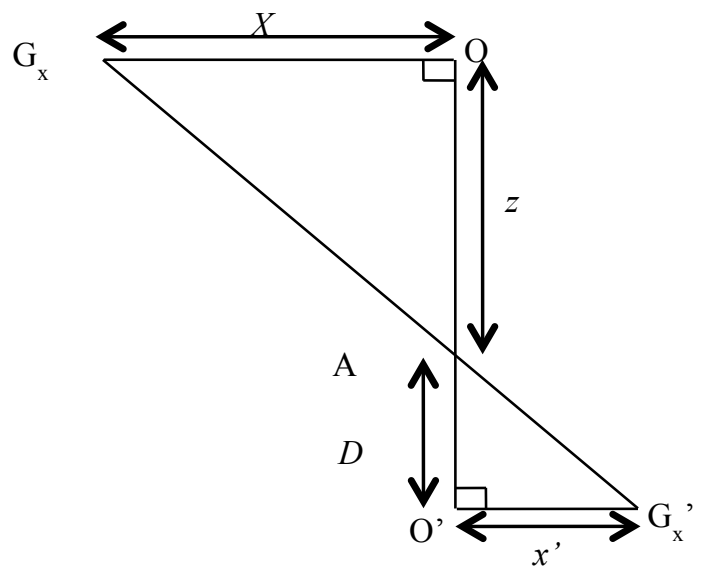

Fig. 5. $\mathrm{OG}_{\mathrm{x}} \mathrm{AO}^{\prime} \mathrm{G}_{\mathrm{x}}$ 'plane.

by the following equations. In addition, it becomes similar about the y-axis direction.

$$
\begin{aligned}
& X=\frac{x^{\prime} z}{D} \\
& Y=\frac{y^{\prime} z}{D}
\end{aligned}
$$

\subsection{Coordinate transformation}

The system converts the coordinate $(X, Y)$ on the view screen into the coordinate $\left(X_{P C}, Y_{P C}\right)$ on the PC. Figure 6 shows the relationships between these two coordinates. Equation (5), (6) gives the coordinate converting method of figure 6 . In this way, the system moves the mouse cursor on the PC screen corresponding to the position of the laser spot

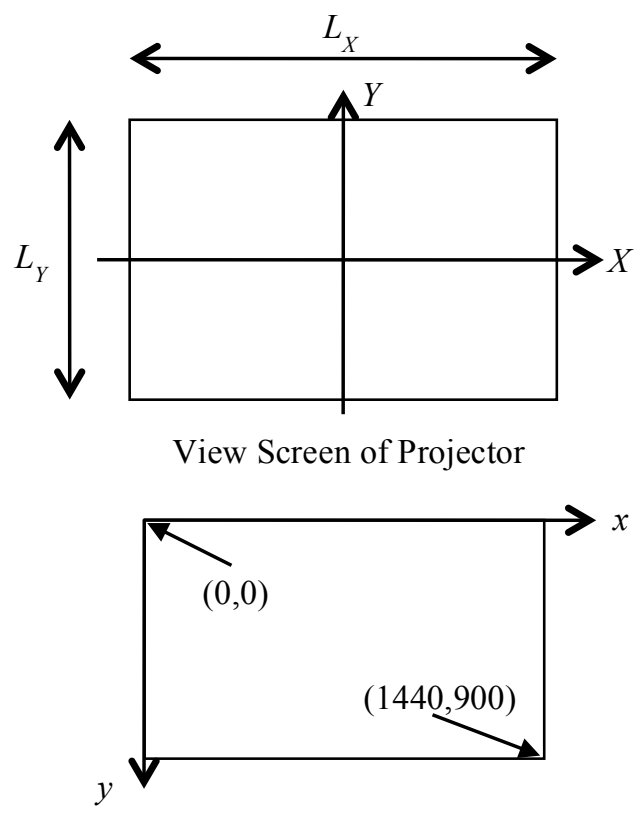

Coordinate system of the PC screen

Fig. 6. Relations of the Coordinate. 
on the view screen.

$$
\begin{aligned}
& X_{P C}=\frac{1440 X}{L_{X}}+720 \\
& Y_{P C}=-\frac{900 Y}{L_{Y}}+450
\end{aligned}
$$

\subsection{Operation of the mouse cursor}

Because this system do not want gives an extra operating device which reducing the operation performance. The proposed laser pointer has two types light source installed. One is normal type laser light source that gives a gazing point to the audience for a good understanding. The other one is modulated light source that the reflect light can be received by PSD sensor to give a coordinate information for PC operation. These two types light source can be switched by control the power source with two buttons installed on the laser pointer. One additional button can rapidly accept by user.

\subsection{Screen drawing system}

We implement a drawing function based on the coordinate information that the system calculated. Consider the coordinate on PC screen as start point when the modulated laser source is emitted, then move the laser light spot on the view screen, if the moving distance is larger than a threshold value, then treat the current point as a end of line interval, then draw a line between theses two points. Repeat this procedure until the modulated light source stop, thus the system draw a picture.

\section{Experiments}

\subsection{Detection range measurement}

In this experiment, the detection range $\mathrm{Lx}, \mathrm{Ly}$ of this system is examined first.

- Project a picture of PC screen to the view screen generated by projector.

- Measure the distance from view screen to the light-receiving module. The measurement range is from 1 meter to 2 meters, and the measuring interval is 0.2 meter.

- Detect the view angle that can measure by PSD in its lens-imaging angle.

Table 1 shows the result of this experiment. By this experiment, we were able to confirm that the angle o

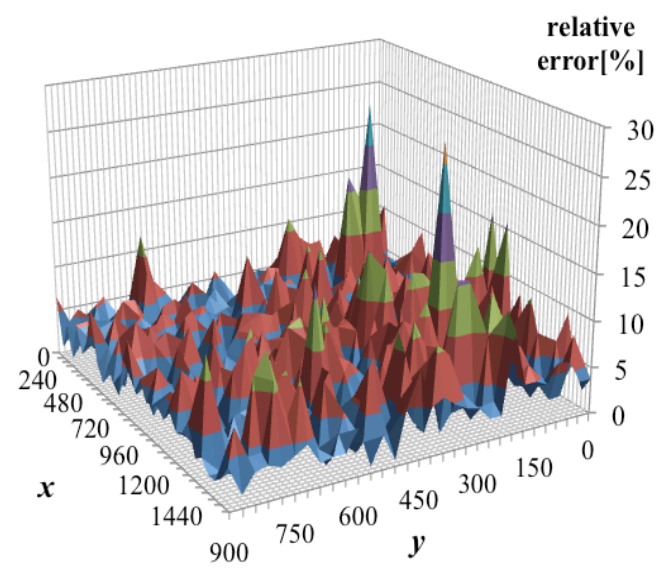

Fig. 7. Relative Error of the Measurement Result. view of this system was approximately 43.6 degrees.

\subsection{Detection accuracy measurement}

The experimental environment is set close to an actually using environment. Then project a PC screen on view screen with a projector in a darkroom and tested it. The power source supply of laser pointer is a pulse modulated one only. We still have not a 2 mode laser point at this time.

In this experiment, it was aimed to evaluate the accuracy of the measurement of the coordinate detection precision of the system. The light-receiving module is set 2 meters far away from the view screen generated by the projector. By acquiring the coordinate of laser irradiation point on the view screen and calculate the relative error with the theoretical value of coordinate, the measurement accuracy was obtained.

Figure 7 shows the measurement results. Although the measurement result is not satisfied enough now, it shows the coordinate of laser spot on the view screen in real time.

Table 1. Measuring Range.

\begin{tabular}{|c|l|l|l|l|l|l|}
\hline Distance $[\mathrm{m}]$ & 1.0 & 1.2 & 1.4 & 1.6 & 1.8 & 2.0 \\
\hline $\mathrm{x}[\mathrm{m}]$ & 0.79 & 1.02 & 1.12 & 1.29 & 1.45 & 1.59 \\
\hline $\mathrm{y}[\mathrm{m}]$ & 0.79 & 0.94 & 1.09 & 1.26 & 1.43 & 1.56 \\
\hline
\end{tabular}

\section{Conclusions}

In this study, we studied detection of laser spot position in real time. According to the result of the experiment, proposed approach could measure the coordinate of the laser spot using a two-dimensional PSD sensor and a lens imaging structure. In addition, we confirmed that the 
accuracy was reasonable to draw picture using a power supply modulated laser pointer. It is possible that the mouse cursor pursued the coordinate of the position of laser spot irradiate on the view screen. Moreover, we could draw picture along the laser point. In this system, we can operate in real time because it didn't use image processing like an existing method. Thereby, we considered that the proposed system could make the presenter to have a presentation smoothly.

In future, it is necessary to reduce the noise comes from the circuit because the PSD output current is very tiny and be influenced easily. We will add other operation such as turn the pages to the system. Therefore, presenter won't get stress when they present with this system.

\section{References}

(1) POPOVICH E : "Presenter Mouse LASER-Pointer Tracking System”, http://www.mpi-inf.mpg.de/ karni/presentermouse/index.htm, access date: 2014.1.31

(2) LUCAS B. D: "An iterative image registration technique with an application to stereo vision", Proc. of International Joint Conference on Artificial Intelligence, 1981

(3) C. Kirstein, H. Muller : "Interaction with a projection screen using a camera-tracked laser pointer", MMM '98 Proceedings of the 1998 Conference on Multi Media Modeling, pp.191, 1998

(4) D. Toyosaki, K. Kai, and M. Iwatsuki: "A Laser Pointer Tracking Mouse with an Internal CCD Camera for Presentations", IEICE Technical Report, ET2005-73, pp. 93-96, 2005

(5) SUKTHANKAR R: "Self-Calibrating Camera-Assisted Presentation Interface", Proceedings of International Conference on Control, Automation, Robotics and Vision (ICARCV), 2000 